\title{
Activation timing of postural muscles of lower legs and prediction of postural disturbance during bilateral arm flexion in older adults
}

\author{
Chie Yaguchi $^{1 *}$, Katsuo Fujiwara ${ }^{2}$ and Naoe Kiyota ${ }^{3}$
}

\begin{abstract}
Background: Activation timings of postural muscles of lower legs and prediction of postural disturbance were investigated in young and older adults during bilateral arm flexion in a self-timing task and an oddball task with different probabilities of target presentation. Arm flexion was started from a standing posture with hands suspended $10 \mathrm{~cm}$ below the horizontal level in front of the body, in which postural control focused on the ankles is important.

Methods: Fourteen young and 14 older adults raised the arms in response to the target sound signal. Three task conditions were used: 15 and 45\% probabilities of the target in the oddball task and self-timing. Analysis items were activation timing of postural muscles (erector spinae, biceps femoris, and gastrocnemius) with respect to the anterior deltoid (AD), and latency and amplitude of the P300 component of event-related brain potential.

Results: For young adults, all postural muscles were activated significantly earlier than AD under each condition, and time of preceding gastrocnemius activation was significantly longer in the order of the self-timing, 45 and 15\% conditions. P300 latency was significantly shorter, and P300 amplitude was significantly smaller under the $45 \%$ condition than under the 15\% condition. For older adults, although all postural muscles, including gastrocnemius, were activated significantly earlier than AD in the self-timing condition, only activation timing of gastrocnemius was not significantly earlier than that of $\mathrm{AD}$ in oddball tasks, regardless of target probability. No significant differences were found between 15 and 45\% conditions in onset times of all postural muscles, and latency and amplitude of P300.

Conclusion: These results suggest that during arm movement, young adults can achieve sufficient postural preparation in proportion to the probability of target presentation in the oddball task. Older adults can achieve postural control using ankle joints in the self-timing task. However, in the oddball task, older adults experience difficulty predicting the timing of target presentation, which could be related to deteriorated cognitive function, resulting in reduced use of the ankle joints for postural control.
\end{abstract}

Keywords: Older adults, Probability, Activation timing of postural muscle, Event-related brain potential, P300, Predictability, Ankle joint

\section{Background}

Deterioration of equilibrium function is considered as a primary cause of falls among older individuals [1]. In several postural tasks needing dynamic equilibrium function, changes in postural control strategy with aging have been reported, particularly in the form of reduced

\footnotetext{
* Correspondence: c_yaguchi@nihoniryo-c.ac.jp

${ }^{1}$ Department of Rehabilitation, Japan Health Care College, 6-17-3

Megumino-nishi, Eniwa 061-1373, Japan

Full list of author information is available at the end of the article
}

involvement of the ankle joints. For instance, when postural disturbance is induced by transient floor translation [1-4] or a load impact against the subject [5], young adults mainly use the ankle joints for postural control $[2,3,5]$, whereas older adults tend to mainly use the hip joints $[1,4,5]$. When older adults voluntarily incline the whole body forward or backward, the lower legs are not inclined in the same direction $[1,6]$. In addition to 
these situations, dynamic postural control is needed during arm movements in daily life.

When arm movements are rapidly executed while standing, the postural muscles of the legs and trunk are automatically activated before the focal muscles of the arms, to moderate any disturbances caused by the arm movements [7]. In older adults, preceding activation of postural muscles is found for the trunk muscles, but is less observable for the thigh muscles, as compared with young adults [8-10]. Furthermore, postural movement during arm movement is smaller at the ankles than at the hips $[11,12]$. These previous studies were performed with arm flexion originating from the side of the body to the horizontal level. Preceding activation of the triceps surae has not been observed during such arm flexion [13] even if young adults start the movement at their own pace (self-timing task) [14]. Cordo and Nashner [15] found that preceding muscle activation was clearly observable in the postural muscles playing the most important roles in balance maintenance. These findings suggest that the focus of postural control would not be on the ankle joints during such arm flexion, and few investigations of postural control patterns in older adults have thus focused on the ankle joints during arm movement.

When arm flexion was performed from a position with the hands suspended in front of the body, preceding activation of the triceps surae has been clearly observed [16-18]. In this posture, immediately after the start of arm flexion, the forward moment caused by the weight of the arms acting on the body should be larger in comparison to the posture with arms at the side of the body. Backward inclination of the whole body pivoting at the ankles is suggested to be a postural movement pattern effectively translating the center of gravity backward $[19,20]$. Thus, in arm flexion from a suspended position, postural control focused on the ankle joints should be needed to resist rapid forward postural disturbance. When young adults performed this arm flexion, activation timing was unaffected by task condition for the erector spinae (ES), but was earlier in a self-timing task than in reaction tasks for leg postural muscles, especially the triceps surae [16]. In the self-timing task, postural disturbance timing was easily predicted, allowing selection of the most suitable postural control pattern. The postural control pattern during arm movement in older adults was thus first investigated using arm flexion from a suspended position in the self-timing task. Older adults were hypothesized to show a postural control pattern focused not on the ankle joints but rather mainly on the hip joints even in situations where postural control focused on the ankles is important.

In an oddball task, where cognitive function is strongly concerned with task execution, a target or non-target stimulus appears repeatedly in random order at a fixed inter-stimulus interval and the participant is required to perform a specific response to the target stimulus [21-23]. In this task, positivity at approximately $300 \mathrm{~ms}$ after target stimulus (P300 component) appears on averaged electroencephalogram (EEG) waveforms recorded from the parietocentral portion [21-23]. P300 is an eventrelated brain potential and reflects cognitive processing, such as evaluation and judgment of sensory stimuli [24] and subsequent context updating [23, 25]. Latency and amplitude of P300 indicate the cognitive processing time and allocation of attention to the processing, respectively [21-23]. With a higher probability of target presentation, prediction of target appearance is reportedly easier [26], and the latency and amplitude of P300 become shorter and smaller [22, 23, 26, 27]. Easier prediction of target presentation timing with a higher probability could thus result in decreased processing time and decreased allocation of attention to the cognitive processing. When arm flexion from a suspended posture is used as a response action in the oddball task, the relationship between changes in cognitive processing and onset timing of postural muscle activation by predictability could be directly investigated. For young adults, with higher probability, the preceding time of the triceps surae activation would be longer, relating to shorter latency and smaller amplitude of P300.

In contrast, changes in P300 amplitude with increasing target probability (from about $20 \%$ to about $80 \%$ ) were reportedly smaller for older adults than for young adults $[26,28]$. This would indicate difficulty in predicting target appearance based on the sequence of target and non-target stimuli and in changing attentional allocation to cognitive processing in proportion to probability. For older adults, the effects of target probability are hypothesized to be less apparent in onset times of postural muscle activation and the latency and amplitude of P300, and onset of the triceps surae activation in oddball tasks would be markedly later than in a selftiming task.

The purpose of this study was to investigate the postural control pattern in older adults focused on the ankle joints during arm movement. For that, older adults performed bilateral arm flexion from a suspended arm position in the self-timing task. Furthermore, to investigate the relationship between anticipatory postural control and cognitive function, this arm movement was carried out in oddball tasks with different probabilities of target presentation.

Our working hypotheses were as follows. First, for young adults, as the probability of target presentation increases, the preceding time of the triceps surae activation with respect to the anterior deltoid (AD) activation would be longer and become closer to that in the self-timing task, with shorter latency and smaller amplitude of P300. Second, for older adults, ES activation would be earlier 
than $\mathrm{AD}$ activation, but the triceps surae activation would not be earlier than AD activation even in the self-timing task. Onset time of postural muscle activation, and latency and amplitude of P300 would be unaffected by target probability in the oddball task, and onsets of the triceps surae would be markedly later than in the self-timing task.

\section{Methods}

\section{Subjects}

Subjects comprised 14 young adults ( 7 men, 7 women) and 14 older adults (11 men, 3 women). Mean values (standard deviation (SD)) for age, height, weight, and foot length were 22.4 (1.9) years, $163.7(5.5) \mathrm{cm}, 57.0$ (8.1) $\mathrm{kg}$, and 24.4 (1.2) $\mathrm{cm}$ for young adults, and 70.3 (5.7) years, $156.3(9.0) \mathrm{cm}, 56.2(7.3) \mathrm{kg}$ and 23.6 (1.4) $\mathrm{cm}$ for older adults. Subject health status was assessed from a questionnaire. All subjects reported no history of neurological or orthopedic impairment and had normal hearing. Older subjects were community-dwelling individuals who could walk independently and perform activities of daily living without assistance. In accordance with the Declaration of Helsinki, all subjects provided informed consent after receiving an explanation of the experimental protocol, which was approved by the ethics committee at Kanazawa University.

\section{Apparatus}

To measure the center of pressure in the anteroposterior direction (CoPap), a force platform (OR6-6; AMTI, USA) was used (Fig. 1). CoPap electronic signals were sent simultaneously to three devices, a computer (PC9801BX; NEC, Japan) to determine CoPap position, another computer (Dimension E521; Dell Japan, Japan) for analysis, and an oscilloscope (DS6612; Iwatsu, Japan) to monitor the results. The onset time of postural muscle activation is influenced by CoPap position just before arm flexion [29]. To control initial CoPap positions, the first computer, which received CoPap data via an analog-to-digital (A/D) converter (PIO9045; I/O-Data, Japan) with a sampling rate of $20 \mathrm{~Hz}$ and 12-bit resolution, generated a buzzing sound when CoPap was located within a range of $\pm 1 \mathrm{~cm}$ of the quiet standing posture (QSP range). Since the SD for CoPap fluctuation during QSP for $60 \mathrm{~s}$ was approximately $0.5 \mathrm{~cm}$ for young healthy adults [30], the QSP range corresponds to \pm 2 SDs of the fluctuation.

Subjects held wooden grips attached beneath a wooden board (17 $\mathrm{cm} \times 37 \mathrm{~cm} \times 1.7 \mathrm{~cm}$; weight, $0.7 \mathrm{~kg})$, with hands fixed to the board by acrylic belts (Fig. 1). The board was suspended by non-extensible metal wires (length, $70 \mathrm{~cm}$ ) from a metal frame set outside the force platform. Total weight of the board was set at 3\% of body weight using free weights. To ensure the subject maintained a standing posture without leaning on the board,

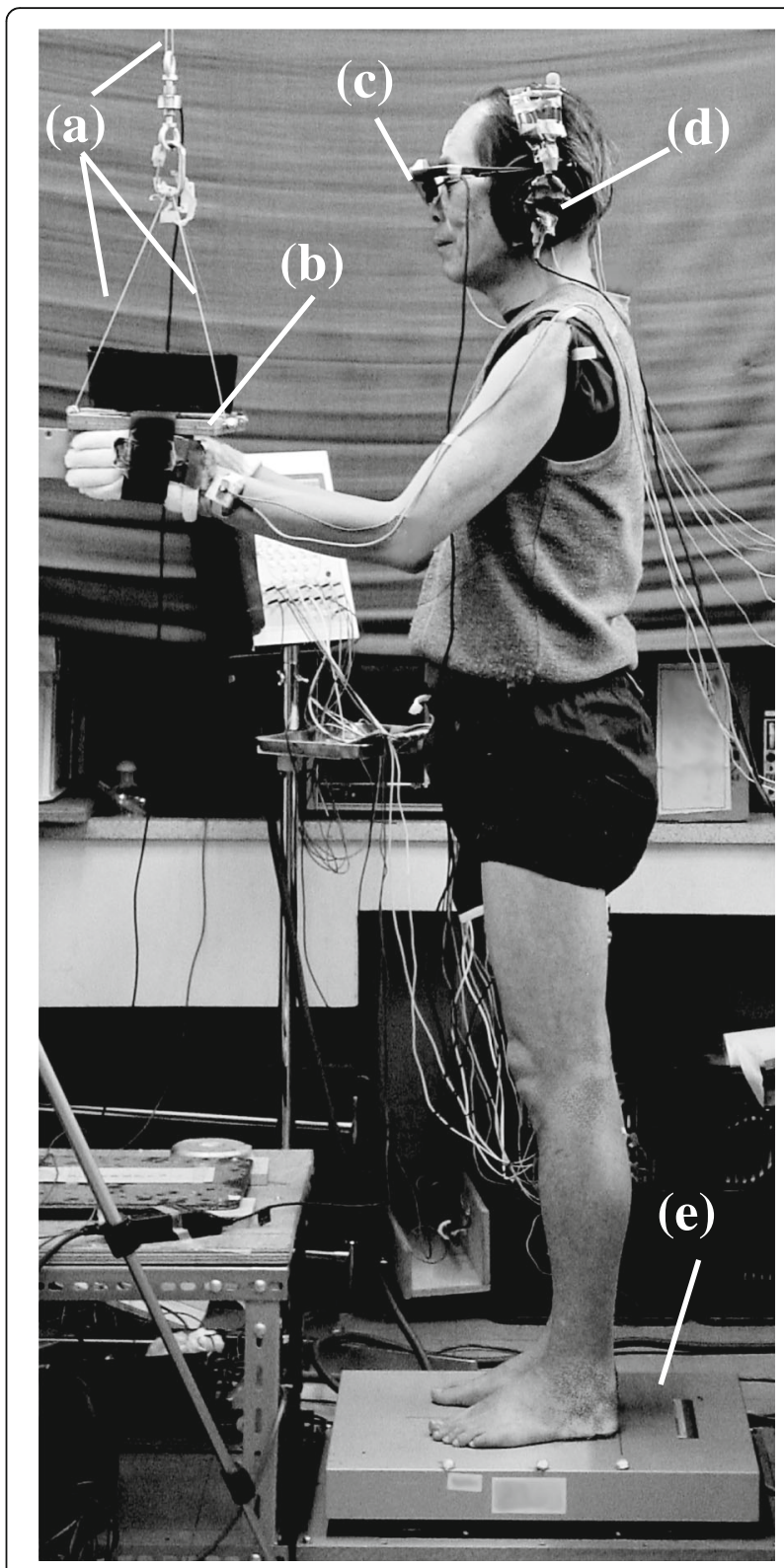

Fig. 1 Experimental setup. a Metal wires. b Wooden board. c Eye-trek face-mounted display. $\mathbf{d}$ Headphones. e Force platform

force applied to the board by the subject was monitored using a load cell (LUB-B-50 KB; Kyowa, Japan) attached to the connection between the frame and wire. The validity of this apparatus for arm flexion with hands suspended has been verified [17]. In the arm flexion task, EEG activity is reported to be minimally contaminated by arm flexion electromyogram (EMG) activity [18].

A fixation point was presented on the center of an eye-trek face-mounted display (FMD011F; Olympus, Japan) (Fig. 1). In oddball tasks, auditory stimuli of 2000 and $1000 \mathrm{~Hz}$ were used as the target and non-target stimuli, respectively. Stimuli were delivered in random 
order via headphones with intensity of $90 \mathrm{~dB}$, duration of $100 \mathrm{~ms}$ and inter-stimulus interval of $2.5 \mathrm{~s}$ (Synax; NEC, Japan). Probabilities of target presentation were set at 15 and $45 \%$.

Surface electrodes (P-00-S; Ambu, Denmark) were used in bipolar derivation to record EMG activity of the following muscles: $\mathrm{AD}$ as a focal muscle for arm flexion; and $\mathrm{ES}$ at the level of the iliac crest, long head of biceps femoris (BF) at the midpoint between the ischial tuberosity and head of the fibula, and medial head of gastrocnemius $(\mathrm{GcM})$ as postural muscles. Electrode location for both $\mathrm{AD}$ and GcM was the midportion of the muscle belly. Since the direction of arm movement (i.e., postural disturbance) was forward only and preceding activation of postural muscles was observed mainly in dorsal muscles [18], only dorsal postural muscles were selected. In the preliminary experiment, we also confirmed that activation of frontal postural muscles was small and did not occur earlier than activation of $\mathrm{AD}$. Electrodes were placed on the right side of the body with an inter-electrode distance of about $3 \mathrm{~cm}$. A ground electrode was placed over the right lateral malleolus. These electrodes were fixed after shaving and cleaning the skin with alcohol.

$\mathrm{Ag}-\mathrm{AgCl}$ cup electrodes (diameter, $8 \mathrm{~mm}$ ) for recording EEG were affixed to the scalp at $\mathrm{Fz}, \mathrm{Cz}$, and $\mathrm{Pz}$ in accordance with the international 10-20 system and referred to linked earlobes. A ground electrode was placed at Fpz. An electrooculogram (EOG) was recorded from a pair of electrodes placed immediately above and below the right eye.

Electrode input impedance was reduced to $<5 \mathrm{k} \Omega$. Signals from electrodes were amplified $(E M G \times 4000$, EEG $\times 10,000, E O G \times 1000)$ and band-pass filtered (EMG $1.6-500 \mathrm{~Hz}$, EEG $0.05-100 \mathrm{~Hz}$, EOG $0.05-30 \mathrm{~Hz}$ ) using an amplifier (EMG BIOTOP-6R12; NEC-Sanei, Japan; EEG and EOG: Synax; NEC, Japan). All electrical signals were sent to the computer for analysis via A/D converters (ADA16-32/2(CB) F; Contec, Japan) with a sampling rate of $1000 \mathrm{~Hz}$ and 16-bit resolution.

\section{Procedure}

All measurements were performed on the force platform while standing barefoot with feet $10 \mathrm{~cm}$ apart and parallel, hands suspended $10 \mathrm{~cm}$ below the shoulder joints, and elbows flexed $60^{\circ}$ (Fig. 1). Subjects were instructed to keep the shoulder muscles as relaxed as possible, not to lean toward the board and to gaze at the fixation point during all measurements. EMG activity in $\mathrm{AD}$ and force applied to the board were monitored by the experimenter and adjusted by oral instruction. Mean CoPap was initially measured 5 times for $10 \mathrm{~s}$ while the subject maintained QSP. The mean value from five measurements was adopted as the QSP position.

Next, bilateral arm flexion trials under the self-timing condition and the oddball task with 15 and 45\% target probability were commenced. Subjects initially maintained CoPap position within the QSP range for at least $3 \mathrm{~s}$ while hearing the buzzing sound, which was then stopped by an experimenter. Under the self-timing condition, within $3 \mathrm{~s}$ of cessation of the buzzing sound, the subject initiated bilateral arm flexion at their own timing. Trials were repeated with a 30-s rest period between trials until 20 trials were accepted. In the oddball task, a 3-min experimental block comprising 72 target or non-target auditory stimuli started $3 \mathrm{~s}$ after cessation of the buzzing sound and the subject was required to respond only to the target stimuli. Experimental blocks were repeated with 3-min seated rest periods until 20 target trials per condition were accepted. Under all conditions, subjects were instructed to flex the arms at maximum speed, stop voluntarily at shoulder level, and maintain this position for $1 \mathrm{~s}$ before returning to the starting position. Trials in which the CoPap position just before arm flexion was beyond the QSP range were excluded from the count of acceptable trials. In the oddball task, trials with eye blinks and excessive muscle-related potentials (voltage on EOG or any EEG electrodes exceeding \pm $100 \mu \mathrm{V}$ ) during the period from $200 \mathrm{~ms}$ before to $800 \mathrm{~ms}$ after target stimulus onset were also excluded. The order of conditions was randomized for each subject. Subjects were given 3 min of seated rest between conditions.

\section{Data analysis}

All data were analyzed while blinded to subject condition, using BIMUTAS II signal analysis software (Kissei Comtec, Japan).

EMGs were analyzed as described below with reference to previous studies [16-18] (Fig. 2). To exclude electrocardiographic and movement artifacts, all EMGs were highpass filtered at $40 \mathrm{~Hz}$ using a seventh-order Butterworth method, then full-wave rectified. Mean and SD of the amplitude for background activity of each muscle was calculated during the period from - 150 to $0 \mathrm{~ms}$ with respect to target onset for $\mathrm{AD}$ and from -300 to $-150 \mathrm{~ms}$ with respect to burst onset of $\mathrm{AD}$ for postural muscles. Burst activation of each muscle was identified when onset was within +200 to $+500 \mathrm{~ms}$ after target onset for $\mathrm{AD}$ and -150 to $+100 \mathrm{~ms}$ with respect to burst onset of $\mathrm{AD}$ for postural muscles, and when the envelope line of the burst activity deviated more than the mean +2 SDs from background activity for at least $50 \mathrm{~ms}$. Burst onset was defined as the time point at which the above deviation began in the EMG wave included in the envelope line. The onset time of postural muscles was defined as the time difference between burst onsets of postural muscles and $\mathrm{AD}$ and presented as a negative value when burst onset of postural muscles preceded AD. Mean values for the onset time of each postural muscle were 


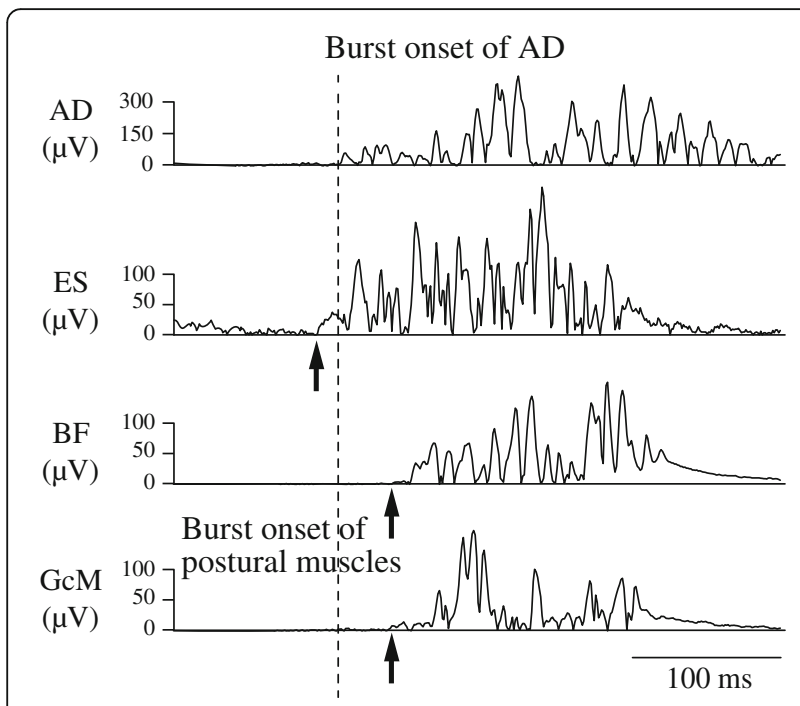

Fig. 2 Representative waveforms of electromyogram data from an older subject performing the oddball task with 15\% target probability. $A D$ anterior deltoid, ES erector spinae, BF biceps femoris, GCM gastrocnemius

calculated separately for all conditions and used as representative values for subjects.

The P300 component is elicited by target stimuli in a discrimination task [25] and show maximum amplitude in the parietal area [31]. In the oddball task, therefore, waveforms for target stimuli recorded from Pz were averaged separately for each condition and analyzed for P300 (Fig. 3). Twenty trials were adopted for each averaging. Averaged epochs extended from $200 \mathrm{~ms}$ before to $800 \mathrm{~ms}$ after target stimulus onset. Mean amplitude during the 200-ms pre-stimulus period was defined as the baseline for averaging. The averaged waveform was smoothed using a $30-\mathrm{Hz}$ low-pass filter. The largest positive peak between 250 and $500 \mathrm{~ms}$ after target stimulus onset was defined as P300 with reference to previous studies [17, 21, 26, 28]. Latency and amplitude of the peak were calculated as the time from onset of target stimulus to the peak and the voltage difference from baseline to peak, respectively.

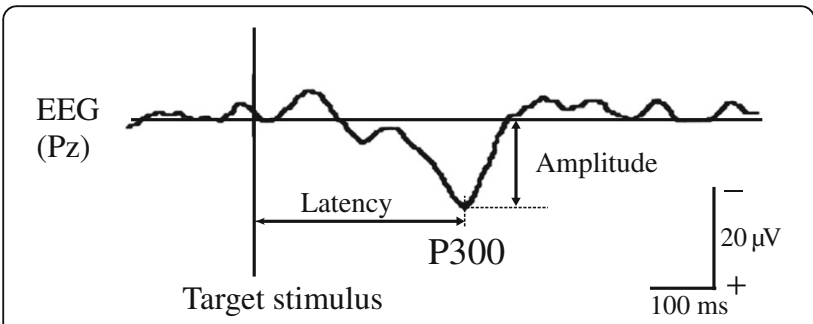

Fig. 3 Representative waveform of P300 component of a young subject performing the oddball task with 15\% target probability. EEG electroencephalogram

\section{Statistical analysis}

The Shapiro-Wilk tests confirmed that all data satisfied the assumptions of a normal distribution. A one-sample $t$ test was used to assess whether burst onset of the postural muscles differed significantly from that of $\mathrm{AD}$. The following analyses were separately used for subject groups. After Levene's tests confirmed that the variances of onset times of each postural muscle were equal in every condition, two-way mixed factorial analysis of variance was used to assess the effects of condition (15\%, 45\%, and self-timing) and muscle (ES, BF, and GcM) on the onset time of postural muscle activation, with repeated measures on the condition factor. Greenhouse-Geisser correction was applied when Mauchly's test of sphericity was not met. When a significant interaction between these effects or a main effect of condition or muscle was shown, post hoc multiplecomparison analyses using Tukey's honestly significant difference were performed separately to assess differences among conditions and muscles. A paired $t$ test was used to assess differences in latency and amplitude of P300 between conditions in oddball tasks (15 and $45 \%)$. Pearson correlations were used to evaluate the magnitude of correlation in oddball tasks between latency or amplitude of P300 and onset time of each postural muscle activation, and between latency and amplitude of P300. For each onset time of postural muscles under each condition, Student's $t$ test was used to investigate differences between young and older adults, after an $F$ test was used to confirm whether variance was equal. Welch's correction was applied when equal variance was rejected. Alpha level was set at $p<0.05$. All statistical analyses were performed using IBM SPSS Statistics version 19 (IBM Japan, Japan).

\section{Results}

Figure 4 shows mean and SD for onset time of postural muscles under each condition. In young adults, all postural muscles were activated significantly earlier than AD under each condition $\left(t_{13}>5.4, p<0.001\right)$. A significant interaction between condition and muscle was found for the onset time of postural muscles in young adults $\left(F_{3.2,61.5}=3.1, p<0.05\right)$. For the significant differences in onset time among conditions, ES showed no differences, BF was earlier in the self-timing condition than in 15 and $45 \%$ conditions $(p<0.01)$, and GcM was earlier in the order of self-timing, 45 and $15 \%$ conditions $(p<0.05)$. In the self-timing condition, onset time tended to be earlier in GcM than in ES ( $p=0.09)$. In 15 and $45 \%$ conditions, no significant differences were found in onset time among muscles. In older adults, activation significantly preceding AD was observed in all muscles under the self-timing condition $\left(t_{13}>3.5\right.$, $p<0.01)$, and in ES and BF under the 15 and $45 \%$ 

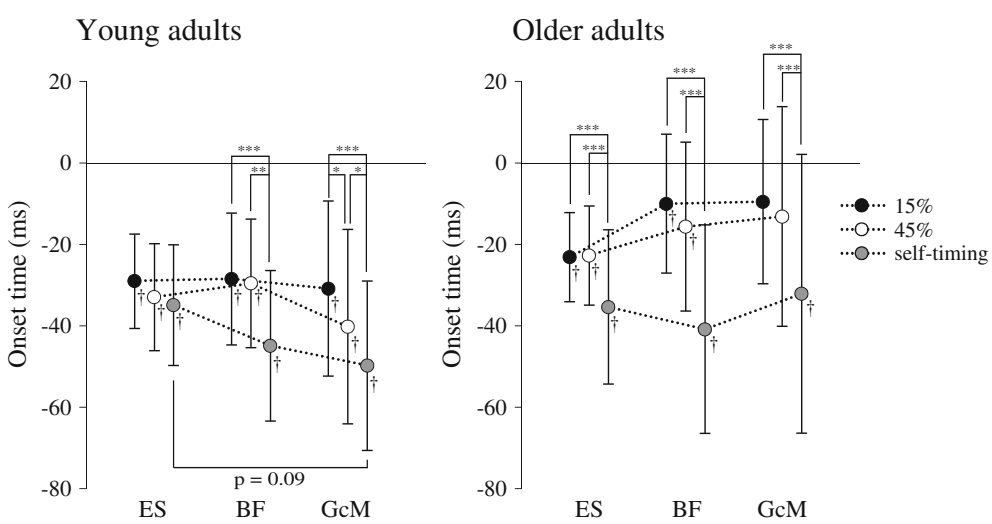

Fig. 4 Means and standard deviations for onset time of postural muscle activation relative to onset of anterior deltoid (AD). ES erector spinae, BF biceps femoris, GCM gastrocnemius. ${ }^{*} p<0.05,{ }^{* *} p<0.01,{ }^{* *} p<0.001$. $†$ Significant differences between burst onsets of $A D$ and each postural muscle $(p<0.05)$

conditions $\left(t_{13}>2.2, p<0.05\right)$. However, preceding activation was not found in GcM under the 15 and $45 \%$ conditions. Only a significant main effect of condition was found for onset time of postural muscle activation in older adults $\left(F_{1.5,58.4}=28.2, p<0.001\right)$. Onset time of all muscle activations was significantly earlier under the self-timing condition than under 15 and $45 \%$ conditions $(p<0.001)$, and no significant difference was found between 15 and $45 \%$ conditions. Only for the onset time of GcM in the self-timing task, variance was significantly larger in older adults than in young adults $\left(F_{13,13}=2.7\right.$, $p<0.05$ ). Onset times of $\mathrm{BF}$ and $\mathrm{GcM}$ in the $15 \%$ condition and ES and GcM in the $45 \%$ condition were significantly later in older adults than in young adults $\left(t_{26}>2.1, p<0.05\right)$. No significant differences were found between young and older adults in onset time of ES in $15 \%$ condition, $\mathrm{BF}$ in $45 \%$ condition, and all postural muscles in the self-timing condition.

Figure 5 shows mean and SD for latency and amplitude of P300 in 15 and 45\% conditions. In young adults, latency and amplitude of P300 were significantly shorter and smaller under the $45 \%$ condition than under the $15 \%$ condition (latency $t_{13}=2.7$, amplitude; $t_{13}=5.6, p<0.05$ ). In older adults, no significant differences in latency or amplitude of P300 were found between conditions.

In both young and older adults, no significant correlations were found between latency or amplitude of P300 and onset time of each postural muscle activation, or between latency and amplitude of P300.

\section{Discussion}

In the self-timing task for young adults, all postural muscles activated significantly earlier than $\mathrm{AD}$, and the preceding time tended to be longer in GcM than in ES (Fig. 4). Older adults also showed preceding activation of all postural muscles, but no significant differences were seen among muscles in terms of preceding time (Fig. 4).
Our result revealed that older adults could use the ankle joints in addition to the trunk and hip joints for postural control during arm flexion with sufficient postural preparation. However, the focus was not wholly on the ankles. No significant differences were found between young and older adults in onset times of all postural muscles in this task. However, only for GcM, variance of onset time was significantly larger in older adults than in young adults.

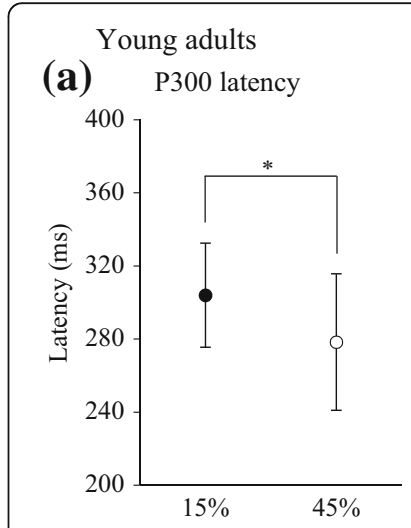

\section{Older adults}

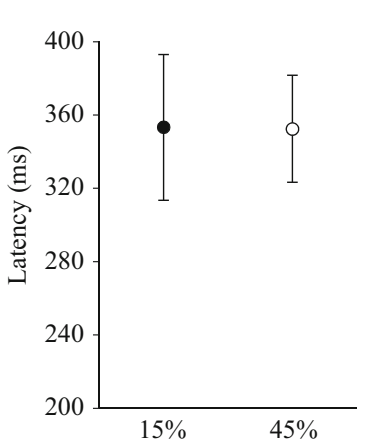

(b) P300 amplitude
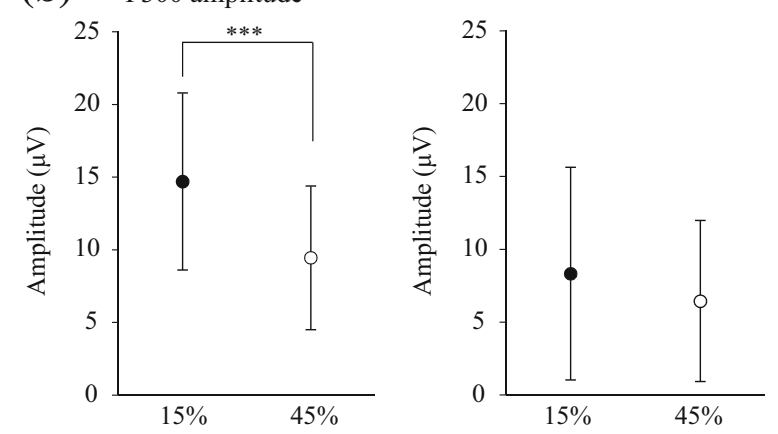

Fig. 5 Means and standard deviations for latency (a) and amplitude (b) of P300. 15\%: oddball task with 15\% target probability; 45\%: oddball task with $45 \%$ target probability. ${ }^{*} p<0.05,{ }^{* *} p<0.001$ 
These results probably indicated that some older adults would show later onset time of GcM. This suggests that age-related deterioration, in the form of reduced use of the ankle joints, would occur in behavior processing for postural control also in the arm movement task, as reported in other postural control tasks [1, 4-6].

In the oddball task for young adults, all postural muscles activated earlier than AD in both 15 and $45 \%$ conditions, and no significant differences in preceding time were seen among muscles (Fig. 4). Onset time of GcM activation in the $45 \%$ condition was significantly earlier than in the $15 \%$ condition but later than in the selftiming task. In this task, cognitive processing of stimuli is necessary in addition to behavior processing and these processes are presumably executed in parallel [32]. These findings suggest that when target probability increases, young adults could predict the presentation timing of target stimuli to some extent, resulting in a longer preceding time of GcM activation. With a higher probability of target presentation, P300 latency became shorter and P300 amplitude became smaller (Fig. 5), consistent with previous findings [22, 23, 26, 27]. Latency and amplitude of P300 indicate the cognitive processing time and allocation of attention to the processing, respectively [21-23]. This indicates that when the target presentation timing could be predicted with higher probability, young adults may perform cognitive processing more rapidly despite less attentional allocation to the processing. This would result in more sufficient postural preparation and earlier onset of activation of the postural muscles in the lower legs. However, changes in onset time of postural muscle activation, especially for GcM, did not show significant correlations with changes in latency or amplitude of P300. Furthermore, no significant correlation was found between changes in latency and amplitude of P300. Predictability may strongly affect cognitive processing time for some subjects and the amount of attentional allocation to this processing for other subjects. Such individual differences would result in an inconsistent relationship between changes in cognitive processing and onset time of postural muscle activation with higher predictability.

For older adults, preceding activation was found in ES and $\mathrm{BF}$, but not in GcM in the oddball task, regardless of the probability. No significant differences in onset time of all postural muscles were seen between 15 and $45 \%$ conditions (Fig. 4). This postural muscle pattern is similar to the pattern when young adults perform arm flexion from a suspended posture in response to a stimulus that appears suddenly [16]. The comparison between postural control patterns in the self-timing and oddball tasks suggests that older adults could not perform postural control primarily using the ankle joints because of deterioration of cognitive function. The latency and amplitude of P300 for older adults also showed no significant differences between 15 and 45\% conditions (Fig. 5). When target stimuli were presented in fixed or random order with a probability of $20 \%$, young adults showed significantly larger P300 amplitude elicited by target stimuli in random order than that in fixed order, but older adults showed no significant differences between P300 amplitudes elicited by targets in fixed and random order [33]. Moreover, compared to young adults, older subjects have shown significantly reduced cortical activation in bilateral superior temporal gyri in an oddball task, which has been interpreted as representing declines in auditory sensory memory and automatic change detection [34]. These findings suggest that older adults would have difficulty predicting the presentation timing of target stimuli based on the sequence of target and non-target stimuli. Furthermore, attentional allocation is reported to be changed according to the significance of the processing to carry out each task [35]. Compared with young adults, postural control for older adults is reported to be strongly impaired by an additional cognitive task during postural control task $[36,37]$. Older adults in the present study might also allocate substantial attention not to behavior processing related to postural control but rather to cognitive processing of stimuli because of difficulty predicting the presentation timing of target stimuli. Therefore, in the oddball task, such difficulty would lead to insufficient postural preparation and reduced use of the ankle joints.

The present study could not demonstrate which agerelated deficits of multiple cognitive aspects, including memory, attention, information processing, and executive function [38], would strongly affect postural controllability, or how these deficits might be related to each other and postural controllability. In future studies, we will set cognitive tasks to separately control for each cognitive aspect and will investigate the relationship between age-related changes in each cognitive function and postural controllability in older adults.

\section{Conclusions}

During arm movement, young adults can achieve sufficient postural preparation in proportion to the probability of target presentation in the oddball task. Older adults can achieve postural control using ankle joints in the self-timing task. However, in the oddball task, older adults experience difficulty predicting the timing of target presentation, which could be related to deteriorated cognitive function, resulting in reduced use of the ankle joints for postural control.

\section{Abbreviations}

A/D: Analog-to-digital; AD: Anterior deltoid; BF: Biceps femoris; CoPap: Center of pressure in the anteroposterior direction; EEG: Electroencephalogram; EMG: Electromyogram; EOG: Electrooculogram; ES: Erector spinae;

GcM: Gastrocnemius; QSP: Quiet standing posture; SD: Standard deviation 


\section{Acknowledgements}

We are grateful to the participants of the present study.

\section{Funding}

Not applicable.

\section{Availability of data and materials}

The datasets used and/or analyzed during the current study available from the corresponding author on reasonable request.

\section{Authors' contributions}

The contribution of each author is as follows: $\mathrm{CY}$ and KF developed the idea of this study, planed the method, directed the experiments, and interpreted the results. NK contributed to the experiments, data analysis, and manuscript preparation. All authors read and approved the final manuscript.

\section{Ethics approval and consent to participate}

In accordance with the Declaration of Helsinki, all subjects provided informed consent after receiving an explanation of the experimental protocol, which was approved by the ethics committee at Kanazawa University.

\section{Consent for publication}

Not applicable.

\section{Competing interests}

The authors declare that they have no competing interests.

\section{Publisher's Note}

Springer Nature remains neutral with regard to jurisdictional claims in published maps and institutional affiliations.

\section{Author details}

${ }^{1}$ Department of Rehabilitation, Japan Health Care College, 6-17-3 Megumino-nishi, Eniwa 061-1373, Japan. ${ }^{2}$ Department of Sports and Health, Kanazawa Gakuin University, 10 Sue-machi, Kanazawa 920-1392, Japan. ${ }^{3}$ Department of Rehabilitation, Japan Health Care College, 6-17-3 Megumino-nishi, Eniwa 061-1373, Japan.

\section{Received: 26 February 2017 Accepted: 14 December 2017}

\section{Published online: 22 December 2017}

\section{References}

1. Horak FB, Shupert CL, Mirka A. Components of postural dyscontrol in the elderly: a review. Neurobiol Aging. 1989;10:727-38.

2. Horak FB, Nashner LM. Central programming of postural movements: adaptation to altered support-surface configurations. J Neurophysiol. 1986:55:1369-81.

3. Fujiwara K, Kiyota N, Maekawa M, Prokopenko SV, Vasilyevna AM. Postural control during transient floor translation while standing with the leg and trunk fixed. Neurosci Lett. 2015;594:93-8.

4. Manchester D, Woollacott M, Zederbauer-Hylton N, Marin O. Visual, vestibular and somatosensory contributions to balance control in the older adult. J Gerontol. 1989:44:M118-27.

5. Kanekar N, Aruin AS. The effect of aging on anticipatory postural control. Exp Brain Res. 2014;232:1127-36.

6. Hyodo A, Fujiwara K, Kiyota N, Toyama H. Postural movement pattern while extreme forward and backward leaning for the elderly. Health Behav Sci. 2017:15:69-73. [In Japanese with English Abstract]

7. Belen'kii VE, Gurfinkel' VS, Pal'tsev El. Elements of control of voluntary movements. Biofizika. 1967;12:135-41.

8. Rogers MW, Kukulka CG, Soderberg GL. Age-related changes in postural responses preceding rapid self-paced and reaction time arm movements. J Gerontol. 1992:47:M159-65.

9. Woollacott MH, Manchester DL. Anticipatory postural adjustments in older adults: are changes in response characteristics due to changes in strategy? J Gerontol. 1993;48:M64-70.

10. Fujiwara K, Toyama H, Asai H, Yaguchi C, Irei M, Naka M, Kaida C. Effects of regular heel-raise training aimed at the soleus muscle on dynamic balance associated with arm movement in elderly women. J Strength Cond Res. 2011;25:2605-15
11. Ai J, Fujiwara K, Asai H, Koshida K, Tomita K, Kawahara N, Tomita H. Postural movement during bilateral arm flexion and deformity of lumbar vertebral bodies related to chronic low back pain in elderly women. Health Behav Sci. 2007:6:9-19.

12. Bleuse S, Cassim F, Blatt JL, Labyt E, Derambure P, Guieu JD, Defebvre L. Effect of age on anticipatory postural adjustments in unilateral arm movement. Gait Posture. 2006:24:203-10.

13. Bouisset $\mathrm{S}$, Zattara M. A sequence of postural movements precedes voluntary movement. Neurosci Lett. 1981;22:263-70.

14. Fujiwara K, Maeda K, Kunita K, Tomita H. Postural movement pattern and muscle action sequence associated with self-paced bilateral arm flexion during standing. Percept Mot Skills. 2007;104:327-34

15. Cordo PJ, Nashner LM. Properties of postural adjustments associated with rapid arm movements. J Neurophysiol. 1982;47:287-302.

16. Fujiwara K, Yaguchi C, Shen X, Maeda K, Mammadova A. Activation timing of postural muscles during bilateral arm flexion in self-timing, oddball and simple-reaction tasks. J Electromyogr Kinesiol. 2011;21:595-601.

17. Shen X, Fujiwara K, Tomita H. Anticipatory postural control associated with bilateral arm flexion and event-related potential in a Kanji Stroop-like task. Clin Neurophysiol. 2009;120:827-33.

18. Fujiwara K, Tomita H, Maeda K, Kunita K. Effects of neck flexion on contingent negative variation and anticipatory postural control during arm movement while standing. J Electromyogr Kinesiol. 2009;19:113-21.

19. Crenna P, Frigo C, Massion J, Pedotti A. Forward and backward axial synergies in man. Exp Brain Res. 1987;65:538-48.

20. Fujiwara K, Yaguchi C. Effects of limiting anterior displacement of the center of foot pressure on anticipatory postural control during bilateral shoulder flexion. J Electromyogr Kinesiol. 2013;23:1460-6.

21. Polich J. P300 in clinical applications: meaning, method, and measurement. Am J EEG Technol. 1991:31:201-31.

22. Luck SJ. An introduction to the event-related potential technique. 2nd ed. London: The MIT Press. 2014;

23. Polich J. Neuropsychology of P300. In: Luck SJ, Kappenman ES, editors. The Oxford handbook of event-related potential components. New York: Oxford University Press; 2012. p. 159-88.

24. Kutas M, McCarthy G, Donchin E. Augmenting mental chronometry: the P300 as a measure of stimulus evaluation time. Science. 1977:197:792-5.

25. Donchin E, Coles MGH. Is the P300 component a manifestation of context updating ? Behav Brain Sci. 1988;11:357-74.

26. Looren de Jong $H$, Kok $A$, Van Rooy JC. Stimulus probability and motor response in young and old adults: an ERP study. Biol Psychol. 1989;29:125-48.

27. Polich J. Task difficulty, probability, and inter-stimulus interval as determinants of P300 from auditory stimuli. Electroencephalogr Clin Neurophysiol. 1987:68:311-20

28. Pfefferbaum A, Ford JM. ERPs to stimuli requiring response production and inhibition: effects of age, probability and visual noise. Electroencephalogr Clin Neurophysiol. 1988;71:55-63.

29. Fujiwara K, Toyama H, Kunita K. Anticipatory activation of postural muscles associated with bilateral arm flexion in subjects with different quiet standing positions. Gait Posture. 2003;17:254-63.

30. Goshima K. Studies on items and normal ranges in stabilometry. Equilibrium Res. 1986;45:368-87. [In Japanese with English Abstract]

31. Simson R, Vaughan HG Jr, Ritter W. The scalp topography of potentials in auditory and visual discrimination tasks. Electroencephalogr Clin Neurophysiol. 1977:42:528-35.

32. Goodin DS, Aminoff MJ. Event-related potentials in the study of sensory discrimination and motor response in simple and choice reaction tasks. J Clin Neurophysiol. 1998;15:34-43

33. Sandman CA, Donnelly JF, O'Halloran JP, Isenhart R. Age-related change in P3 amplitude as a function of predictable and unpredictable rare events. Int J Neurosci. 1990:52:189-99.

34. Cheng $\mathrm{CH}$, Baillet $\mathrm{S}$, Hsiao FJ, Lin YY. Effects of aging on neuromagnetic mismatch responses to pitch changes. Neurosci Lett. 2013;544:20-4.

35. Lavie N. Perceptual load as a necessary condition for selective attention. J Exp Psychol Hum Percept Perform. 1995:21:451-68.

36. Woollacott M, Shumway-Cook A. Attention and the control of posture and gait: a review of an emerging area of research. Gait Posture. 2002;16:1-14.

37. Maki BE, Mcllroy WE. Cognitive demands and cortical control of human balance-recovery reactions. J Neural Transm. 2007;114:1279-96.

38. Hedden T, Gabrieli JD. Insights into the ageing mind: a view from cognitive neuroscience. Nat Rev Neurosci. 2004;5:87-96. 